\title{
Recent investigations on some cubic Laves phase compounds of rare earth and transition metals
}

\author{
K V S RAMA RAO \\ Department of Physics, Indian Institute of Technology, Madras 600036, India
}

Abstract. Pseudobinary alloy, $\mathrm{Tb}_{0.27} \mathrm{Dy}_{0.73} \mathrm{Fe}_{2-\delta}$, belonging to $\mathrm{C}-15$ cubic Laves phase having $\mathrm{MgCu}_{2}$-type structure, possesses large magnetostriction a $\mathrm{d}$ l high magnetomechanical coupling coefficient. The advantages of this material over existing piezoelectric materials, particularly for SONAR applications, are highlighted. Recent results on the influence of cobalt $(\mathrm{Co})$ on magnetic and magnetomechanical properties of $\mathrm{Tb}_{0.27} \mathrm{Dy}_{0.73} \mathrm{Fe}_{2}$ are discussed.

Keywords. Laves phase compounds; magnetostriction; magnetomechanical coupling coefficient.

\section{Introduction}

Piezoelectric and magnetostrictive materials are employed as active elements in transducers. For SONAR applications, piezoelectric materials such as barium titanate $\left(\mathrm{BaTiO}_{3}\right)$ and lead zirconate titanate (PZT-4) are widely used since they possess higher electromechanical coefficient $\left(k_{33} \approx 0.6\right)$ and large strain $(\lambda)$ compared to conventional magnetostrictive materials such as $\mathrm{Ni}$ and $\mathrm{Ni}-\mathrm{Co}$ alloys $\left(k_{33} \approx 0 \cdot 30\right)$ (Sherman 1975; Belov et al 1983).

The distance at which sound waves travel from a SONAR depends on the frequency of operation. The attenuation of sound waves in water increases rapidly with increase in frequency. Therefore, for SONAR applications, it is desirable to have as low a frequency as possible to achieve long ranges. However, the realization of very low frequency $(<1 \mathrm{kHz}$ ) SONARS was hindered by large weight and size considerations using the existing PZT-4. Above all, piezoelectric materials are capacitive in nature and practical problems arise when the transducers are driven at low frequencies (Oswin 1988; Sewell and Kuhln 1988). Since magnetostrictive transducers are inductive (low impedance) devices, the above problem can be easily overcome.

During the early sixties, large magnetostriction was measured at low temperatures on the pure elements of rare-earth (RE) metals. The ordering temperatures for RE were well below room temperature (RT). However, it was possible to retain the large magnetostriction above $R T$ by alloying the $R E$ elements with transition metals (TM) having high ordering temperatures. The RE and TM form a series of intermetallic compounds and among these, $\mathrm{REFe}_{2}$ possesses a large Curie temperature and magnetostriction. These materials belong to the cubic Laves phase compounds with $\mathrm{MgCu}_{2}$-type structure. $\mathrm{TbFe}_{2}$ and $\mathrm{SmFe}_{2}$ have the largest $\mathrm{RT}$ magnetostriction with a large magnetocrystalline anisotropy, arising from RE sublattice. Large magnetic fields of the order of 3-4 tesla are required to achieve these large saturation magnetostriction which hinder practical applications (Clark 1980).

Large variations in the magnitude and sign of magnetocrystalline anisotropy constant $\left(K_{1}\right)$ and magnetostriction were observed in $\mathrm{REFe}_{2}$ compounds and these are given in table 1. It is seen that magnetostriction of $\mathrm{TbFe}_{2}, \mathrm{DyFe}_{2}$ and $\mathrm{HoFe}_{2}$ 
are of the same sign (positive). The anisotropy constant is negative in $\mathrm{TbFe}_{2}$ but positive in $\mathrm{DyFe}_{2}$ and $\mathrm{HoFe}_{2}$. Because of the similarity in magnetostriction sign and dissimilarity in anisotropy constants, Clark (1980) prepared pseudobinary compounds of $\mathrm{TbFe}_{2}-\mathrm{DyFe}_{2}$ and achieved anisotropy compensation in $\mathrm{Tb}_{0.27} \mathrm{Dy}_{0.73} \mathrm{~F}_{2}$. Similarly minimum anisotropy was observed by him in $\mathrm{Ho}_{0.85} \mathrm{~Tb}_{0.15} \mathrm{Fe}_{2}$. Both compounds could be saturated at low magnetic fields at $R T$. Of these, $\mathrm{Tb}_{0.27} \mathrm{Dy}_{0.73} \mathrm{Fe}_{2}$ was found to be a good candidate for SONAR and other applications as it possesses a larger magnetostriction.

Several groups are working towards improving the material properties such as magnetostriction, strength etc by changing the concentration of $\mathrm{Fe}$ (Branwood et al 1986; Abell et al 1986). Microstructural studies (Abell and Lord 1986) suggest that excess RE in non-stoichiometry compounds form a network at the grain boundaries. Since pure $R E$ is ductile in nature, this increases the strength of the material. In addition, magnetostrictive properties also depend on the preparation method of the material (Branwood et al 1986).

The properties of $\mathrm{Tb}_{0.27} \mathrm{Dy}_{0.73} \mathrm{Fe}_{2}$ are compared with those of the existing PZT in table 2. The low Young's modulus and hence a low sound velocity in these materials made it a good candidate for low-frequency SONARs. The other advantage is a considerable reduction in SONAR size (TERFENOL 1986).

In $\mathrm{REFe}_{2}$ compounds, magnetic properties vary considerably by the substitution

Table 1. Magnetostriction and anisotropy constants in $\mathrm{REFe}_{2}$ compounds at $\mathrm{RT}^{*}$.

\begin{tabular}{lcc}
\hline Material & $i_{s}\left(\times 10^{-6}\right)$ & $K_{1}\left(\times 10^{-4} \mathrm{ergs} / \mathrm{cm}^{3}\right)$ \\
\hline $\mathrm{SmFe}_{2}$ & -1560 & - \\
$\mathrm{TbFe}_{2}$ & +1753 & -6300 \\
$\mathrm{DyFe}_{2}$ & +433 & +2450 \\
$\mathrm{HoFe}_{2}$ & +80 & +590 \\
$\mathrm{ErFe}_{2}$ & -299 & -310 \\
$\mathrm{TmFe}_{2}$ & -123 & -43 \\
$\mathrm{Ho}_{0.85} \mathrm{~Tb}_{0.15} \mathrm{Fe}_{2}$ & +325 & -22 \\
$\mathrm{~Tb}_{0.27} \mathrm{Dy}_{0.73} \mathrm{Fe}_{2}$ & +1000 & -60 \\
\hline
\end{tabular}

* taken from Clark (1980)

Table 2. Comparison of the properties of $\dot{\mathrm{REFe}}_{2}$ with PZT.

\begin{tabular}{lcc}
\hline & PZT & REFe $_{2}$ \\
\hline Strain $\left(\times 10^{-6}\right)$ & $100-300$ & $1400^{+}-2000^{*}$ \\
Coupling factor & 0.60 & $0.6^{+}-0.8^{*}$ \\
Density $\left(\times 10^{3} \mathrm{~kg} / \mathrm{m}^{3}\right)$ & $7 \cdot 5$ & $9 \cdot 25$ \\
Young's modulus $\left(\times 10^{10} \mathrm{~N} / \mathrm{m}^{2}\right)$ & $7 \cdot 3$ & $4.5^{+}-2 \cdot 5^{*}$ \\
Sound speed $(\mathrm{m} / \mathrm{s})$ & 3200 & $2500^{+}-1800^{*}$ \\
Electromechanical & & \\
energy density $\left(\mathrm{J} / \mathrm{m}^{3}\right)$ & 670 & $5300^{+}-18000^{*}$ \\
\hline
\end{tabular}

${ }^{+}$arc/induction melted; $*$ free float zone 
of RE or TM metals. The nature of magnetic interactions can be obtained by studying the changes in magnetic properties when one TM is replaced by another (Bushow 1980; Dhilsha and Rama Rao 1990, 1993; Dhilsha et al 1991; Senthil Kumar et al 1993). Of the TM metals, Co behaves unusually and acquires induced magnetic moment. Some of the recent investigations carried out on the effect of $\mathrm{Co}$ on the magnetic and magnetomechanical properties of $\mathrm{Tb}_{0.27} \mathrm{Dy}_{0.73} \mathrm{Fe}_{2}$ system (Dhilsha and Rama Rao 1993) are discussed here.

\section{Preparation of samples}

The samples for measurement of magnetostriction and magnetomechanical coupling coefficient $\left(k_{33}\right)$ were prepared by two methods. In the first, alloys were prepared by melting the constituent rare earth elements of $99.9 \%$ purity and transition metals of $99.99 \%$ purity in an arc furnace under argon atmosphere. The ingots were melted several times to ensure homogeneity. The alloys were then cast in the form of rods and annealed in vacuum at $1173 \mathrm{~K}$ for 10 days. These are designated as $\mathrm{A}$ samples.

In the second method, the constituent elements were initially melted in an arc furnace. The rods obtained after several meltings were remelted in evacuated quartz tubes using an induction furnace. The samples were then zone-melted using an induction furnace. The r.f. coil was fixed in a position and the samples lowered at $30 \mathrm{~cm}$ per hour. The rods thus obtained were annealed in vacuum at $1173 \mathrm{~K}$ for five days. These are designated as I samples.

The samples were found to have formed in single phase with $\mathrm{MgCu}_{2}$-type structure by taking powder $\mathrm{X}$-ray diffractograms (using $\mathrm{CuK}_{\alpha}$ radiation). The lattice parameters were determined by least-squares refinement. Magnetization measurements were carried out from $77 \mathrm{~K}$ to above Curie temperature $\left(T_{c}\right)$ using a PAR-155 vibrating sample magnetometer (VSM) up to an applied field of $10 \mathrm{kOe}$.

Optical micrographs of both A and I samples were taken using a Leitz Loborlux $12 \mathrm{ME}$ microscope.

Magnetostriction was measured using electrical strain gauges and Wheatstone network. Temperature-compensated $120 \Omega$ Karma foil gauges with negligible magnetoresistance (from Micro Measurements Group, USA) were used. The gauge was attached to the sample with a cynoacrylic cement (M-bond 200 or Anabond 202). A $10 \mathrm{~cm}$ Bruker electromagnet was used for generating the dc magnetic field and the measurements were performed up to an applied field of $\approx 5 \mathrm{kOe}$.

The dynamic strain coefficient $\left(d_{33}\right)$ measurements were carried out at a frequency of $70 \mathrm{~Hz}$ with an excitation field $\left(H_{\mathrm{ac}}\right)$ of 2 Oe. $d_{33}$ was measured using the same strain gauges as those used for static magnetostriction.

The samples were subjected to both ac and dc magnetic fields simultaneously along the axis of the rod. The induced voltages across the gauge due to periodic changes in gauge resistance (strain-induced signals) were measured using a lock-in amplifier. $d_{33}$ was determined using the formula

$$
d_{33}=(\delta l / l)_{\mathrm{ac}} / H_{\mathrm{ac}}
$$

where $(\delta l / l)$ is the incremental strain. The measurements were carried out for different applied fields.

Details of the magnetomechanical coupling coefficient $\left(k_{33}\right)$ measured by the three- 
parameter method have been described elsewhere (Dhilsha et al 1991; Dhilsha 1992; Dhilsha and Rama Rao 1993).

The Young's modulus of the samples $\left(1 / S_{33}\right)$ was measured by an indirect method in which mechanical oscillations were set up in the sample through magnetomechanical coupling itself and the resonance/antiresonance frequencies were measured. The experimental set-up used is based on a method developed by Savage et al (1975).

A coil was sparsely but tightly wound around the sample and this acts as a pick-up coil to measure induction $B$ and as a mount to allow the sample to vibrate freely. This sub-assembly was placed in a second coil which generates an ac exciting field (2Oe) and the whole assembly was placed in the electromagnet. The frequency was varied from 0 to $100 \mathrm{kHz}$. The voltage (in phase with the reference drive current) induced in the pick-up coil was measured using a PAR lock-in amplifier and was plotted as a function of frequency. The experiments were repeated for different bias (dc) fields. The variation of the pick-up voltage with frequency is in principle analogous to the plot of the modulus of the impedance versus frequency. The resonance and antiresonance frequencies were determined from the plot. $S_{33}^{B}$, which is $S_{33}$ at constant induction, was calculated using the formula,

$$
S_{33}^{B}=1 /\left(4 L^{2} \rho f_{a}^{2}\right),
$$

where $L$ is the length of the sample, $\rho$ the density of the material and $f_{a}$ the antiresonance frequency.

\section{Results and discussion}

The variation of lattice parameter of $\mathrm{Tb}_{0.27} \mathrm{Dy}_{0.73} \mathrm{Fe}_{2-x} \mathrm{Co}_{x}(x=0 \cdot 0,0 \cdot 5,1.2$ and 2) with $x$ showed a deviation from Vegard's law (Dhilsha and Rama Rao 1993), similar to what was reported for $\mathrm{RE}\left(\mathrm{Fe}_{1-x} \mathrm{Co}_{x}\right)_{2}$ (Dhilsha and Rama Rao 1990; Korolev et al 1990) and $\mathrm{Ho}_{0.85} \mathrm{~Tb}_{0.15} \mathrm{Fe}_{2-x} \mathrm{Ni}_{x}$ (Senthil Kumar et al 1993). Such a behaviour has been attributed to magnetovolume (MV) effects and charge transfer (Eriksson et al 1989; Senthil Kumar et al 1993).

The variations of $T_{c}$ magnetic moment at $\mathrm{RT}$ and $77 \mathrm{~K}$ with increasing Co concentration are given in figure 1 . An increase in $T_{c}$ with $x$ was observed for low Co concentrations suggesting that the TM-TM and RE-TM interactions are stronger in $x=0.5$ sample than in $\mathrm{Tb}_{0.27} \mathrm{Dy}_{0.73} \mathrm{Fe}_{2}$. However, the RT magnetic moment decreases with increasing Co content. Since these compounds are ferrimagnets with RE sublattice moment dominant, the decrease in magnetization must be due to: (a) decreasing dominant $\mathrm{RE}$ sublattice moment $m_{\mathrm{RE}}$ or (b) a constant or an increasing $\mathrm{RE}$ moment accompanied by a sufficient increase in the antiparrallel $\left(\mathrm{Fe}_{1-x} \mathrm{Co}_{x}\right)$ sublattice moment $m_{\mathrm{TM}}$. A decrease in $m_{\mathrm{RE}}$ is unlikely for the Co compounds with small amounts of $x$ because of their increased values of $T_{c}$. Since small amounts of Co in $\left(\mathrm{Fe}_{1-x} \mathrm{Co}_{x}\right)$ compounds can cause an increase in moment, case (b) is presumed to be more likely.

The optical micrographs taken on $\mathbf{A}$ and I samples suggest that A samples contain randomly oriented grains. The I samples are found to have elongated grains along the growth axis.

Static and dynamic magnetostriction were measured at $\mathrm{RT}$ on rods up to an applied magnetic field of $\approx 5 \mathrm{kOe}$ in both $A$ and I samples. Samples were of length $\approx 5 \mathrm{~cm}$. 


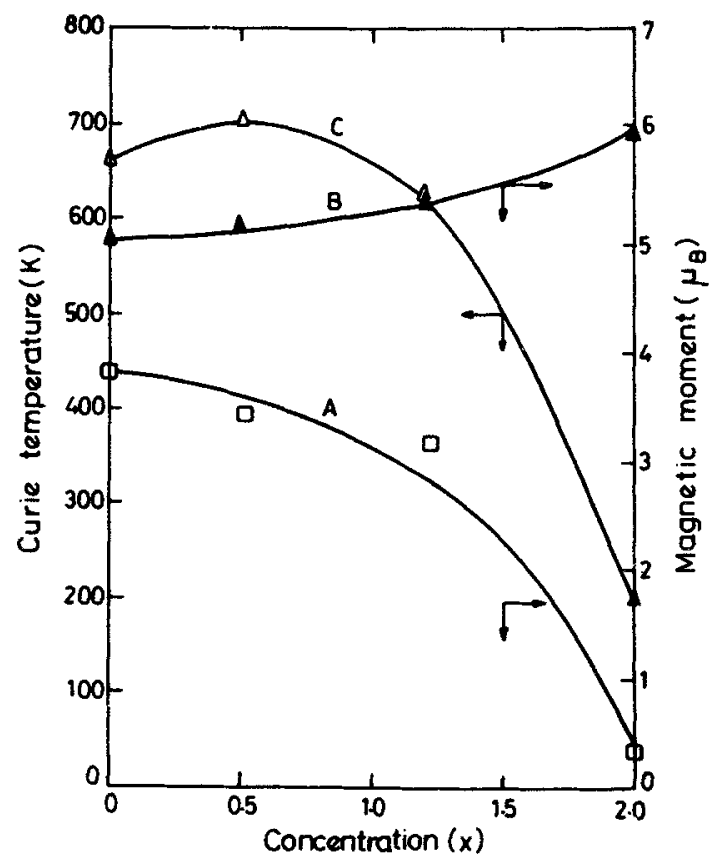

Figure 1. Variation of (A) magnetic moment at RT, (B) magnetic moment at $77 \mathrm{~K}$ and (C) Curie temperature for different Co concentration of $\mathrm{Tb}_{0.27} \mathrm{Dy}_{0.73} \mathrm{Fe}_{2-x} \mathrm{Co}_{x}$.

Table 3. Magnetostriction $(\lambda)$ values at $5.2 \mathrm{kOe}$ for $\mathrm{Tb}_{0.27} \mathrm{Dy}_{0.73} \mathrm{Fe}_{2-x} \mathrm{Co}_{x}$.

\begin{tabular}{lcc}
\hline & \multicolumn{2}{c}{$\lambda\left(\times 10^{-6}\right)$} \\
\cline { 2 - 3 } $\begin{array}{l}\text { Composition } \\
(x)\end{array}$ & A & I \\
& sample & sample \\
\hline 0.0 & $930 \pm 50$ & $1350 \pm 50$ \\
0.5 & $540+20$ & $725 \pm 30$ \\
1.2 & $430 \pm 20$ & $310 \pm 10$ \\
\hline
\end{tabular}

The magnetostriction $(\lambda)$ values obtained at $5 \cdot 2 \mathrm{kOe}$ for $\mathrm{A}$ and I samples of $\mathrm{Tb}_{0.27}$ $\mathrm{Dy}_{0.73} \mathrm{Fe}_{2-x} \mathrm{Co}_{x}$ are given in table 3. $\lambda$ values decrease with increasing Co concentration in $A$ and $I$ samples. However in sample $I, \lambda$ values are higher than that in $A$ sample for $x=0$ and $x=0.5$. This is attributed to a better grain orientation in the induction melted (zoned) samples. Grain orientation also resulted in an increase in the magnetomechanical coupling coefficient $\left(k_{33}\right)$ of I samples compared to that of A samples (Dhilsha and Rama Rao 1993). But a low value of $\lambda$ was observed in I samples with a composition $x=1 \cdot 2$. In $\mathrm{RECo}_{2}$, the sign of $\lambda_{100}$ is reported to be negative with values comparable to that of $\mathrm{REFe}_{2}$ (Lee and Pourarian 1976). In $\mathrm{REFe}_{2}$ compounds, it was found that the $\lambda$ values are dependent on the easy direction of magnetization viz. $\lambda_{111} \gg \lambda_{100}$ (Clark 1980). Therefore, a decrease in magnetostriction values with increasing Co concentration can be due to a change in the easy 


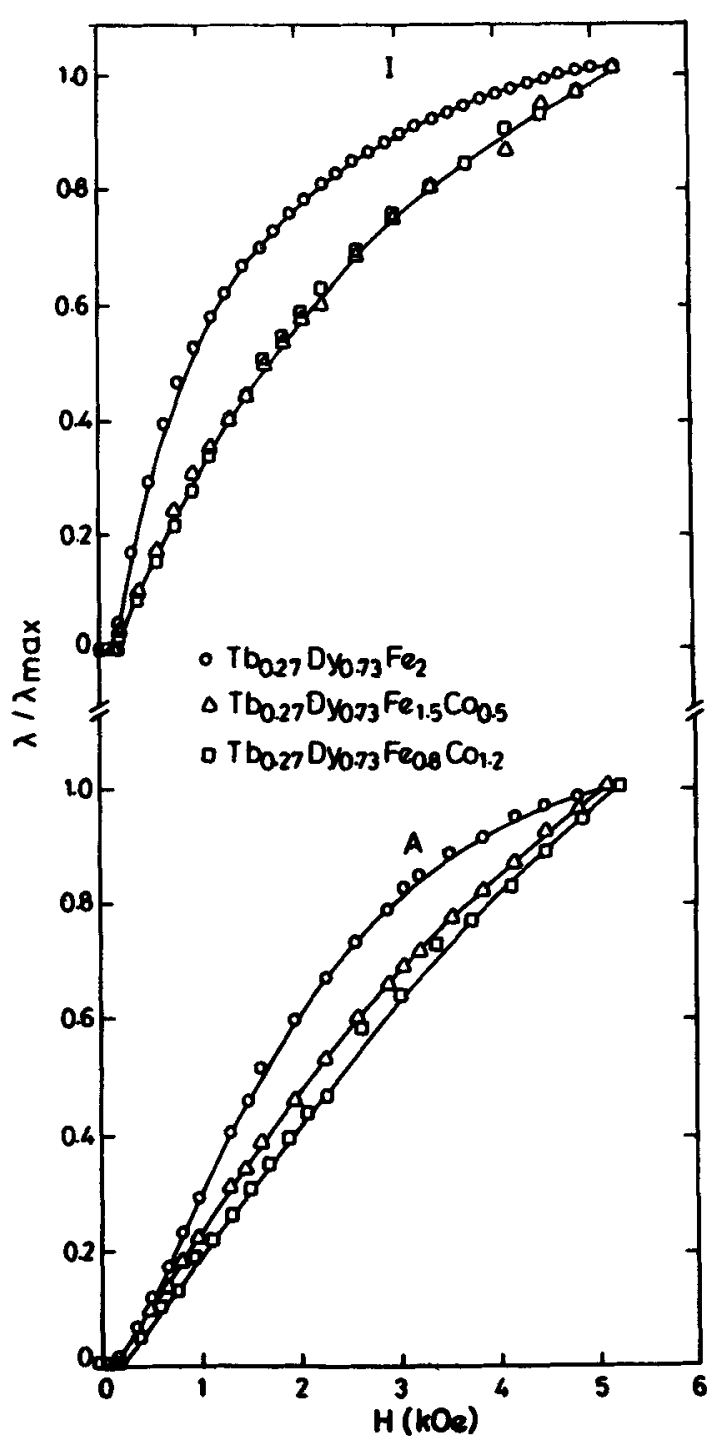

Figure 2. Normalized magnetostriction as a function of applied field for A and I samples of $\mathrm{Tb}_{0.27} \mathrm{Dy}_{0.73} \mathrm{Fe}_{2-x} \mathrm{Co}_{x}$

direction of magnetization as well as a negative contribution of $\lambda$ from Co site (Lee and Pourarian 1976).

The variation of normalized $\left(\lambda / \lambda_{\max }\right)$ versus applied field for $A$ and I samples of $\mathrm{Tb}_{0.27} \mathrm{Dy}_{0.73} \mathrm{Fe}_{2-x} \mathrm{Co}_{x}$ is given in figure 2. Since the aspect ratio of the rods was greater than 5, the demagnetizing effects are neglected (Cullity 1972). It can be seen from figure 2 that I samples tend to saturate at lower bias fields compared to A samples. This low anisotropy of I samples is attributed to preferred grain orientation in these samples. The $\lambda$ values and field dependence of $\lambda$ are dependent on the state of magnetization, original domain configuration and preferred orientation (Jiles and Hariharan 1990). 
An important feature of $\lambda / \lambda_{\max }$ versus field curves for I samples for $x=0.5$ and 1.2 is that they behave in a similar way and indicate a strong dependence of $\lambda$ on the applied field compared to $x=0 \cdot 0$. This also indicates a change in the easy direction of magnetization for $x=0.5$ composition. From Mössbauer (Annapoorni and Rama Rao 1990) and torque magnetometry (Sato et al 1986) it was inferred that the easy direction of magnetization at $\mathrm{RT}$ in $\mathrm{Tb}_{0.27} \mathrm{Dy}_{0.73} \mathrm{Fe}_{2}$ is [111]. In the case of $x=0.5$ and $1 \cdot 2$, the $\lambda$ values were low compared to $x=0.0$. Furthermore, the plots of $x=0.5$ and 1.2 show a linear behaviour compared to $x=0.0$. The linear dependence of $\lambda$ is typical when [100] is the easy direction of magnetization (Kendall and Piercy 1990).

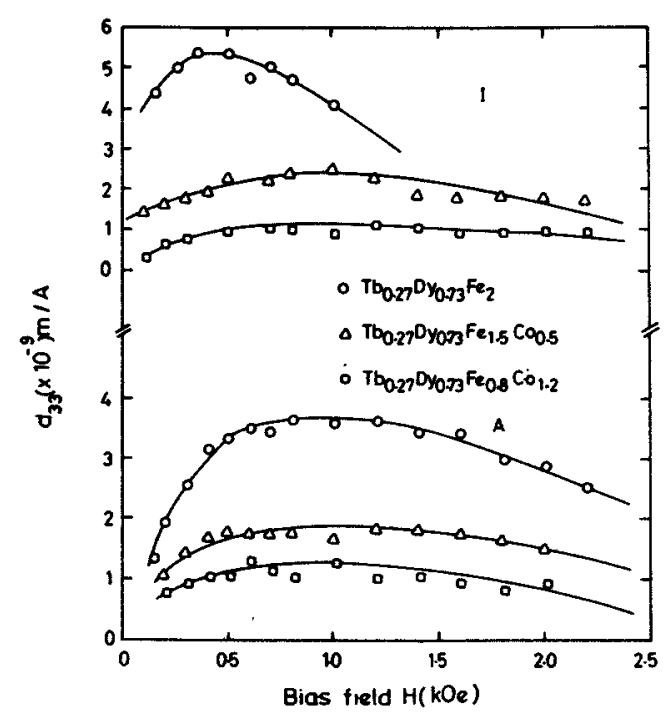

Figure 3. Variation of $d_{33}$ values for $\mathrm{A}$ and $\mathrm{I}$ samples of $\mathrm{Tb}_{0 \cdot 27} \mathrm{Dy}_{0 \cdot 73} \mathrm{Fe}_{2-x} \mathrm{Co}_{x}$.

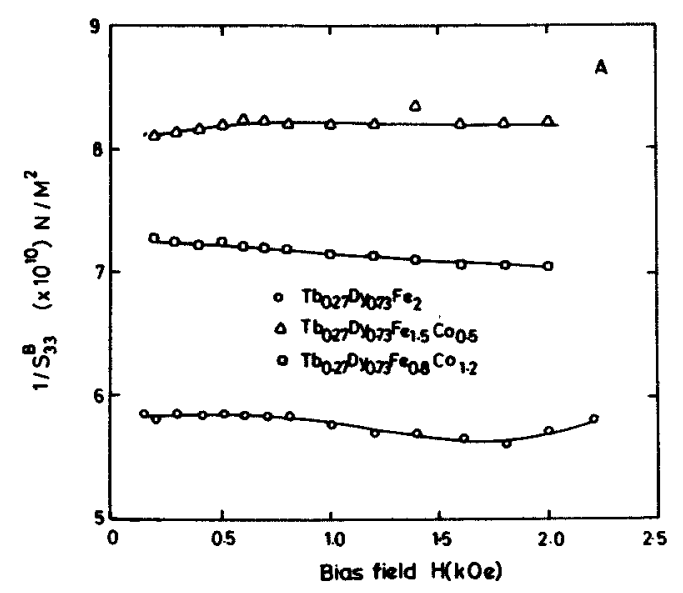

Figure 4. Reciprocal of elastic compliance constant at constant flux density $\left(S_{33}^{B}\right)$ for $\mathrm{A}$ samples of $\mathrm{Tb}_{0 \cdot 27} \mathrm{Dy}_{0.73} \mathrm{Fe}_{2-x} \mathrm{Co}_{x}$. 


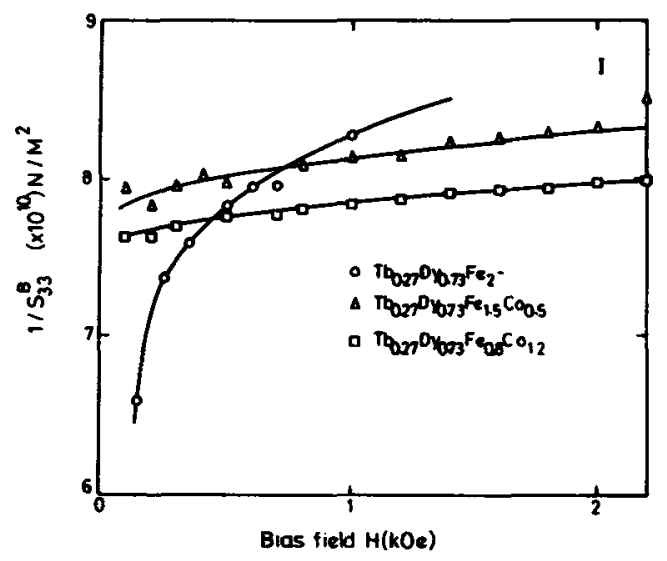

Figure 5. Reciprocal of elastic compliance constant at constant flux density $\left(S_{33}^{B}\right)$ for I samples of $\mathrm{Tb}_{0-27} \mathrm{Dy}_{0.73} \mathrm{Fe}_{2-x} \mathrm{Co}_{x}$.

A similar change in the easy direction of magnetization from [111] to [110] was also observed in the $\mathrm{Ho}_{0.85} \mathrm{~Tb}_{0.15} \mathrm{Fe}_{2-x} \mathrm{Co}_{x}$ system (Dhilsha et al 1991).

The variation of dynamic strain coefficient $\left(d_{33}\right)$ as a function of applied bias field in $A$ and $I$ samples of $\mathrm{Tb}_{0.27} \mathrm{Dy}_{0.73} \mathrm{Fe}_{2-x} \mathrm{Co}_{x}$ is shown in figure 3. The dynamic strain coefficient $\left(d_{33}\right)$ can be seen to be a maximum at a particular bias field. A shift of this maximum towards higher bias fields is indicative of an increase in the magnetocrystalline anisotropy in the system with increasing Co concentration. The magnetization process in a system is related to the easy direction of magnetization. The difference in the behaviour of $d_{33}$ vs $H$ curves for $x=0$ compared to $x=0.5$ and 1.2 suggests that the variation of $d_{33}$ against $H$ is related to the magnetization process in a system (Cullity 1972). A constant value of $d_{33}$ is seen in the case of $x=0.5$ and 1.2 whereas it shows a clear peak for $x=0$. This also indicates a change in the easy direction of magnetization with an increase in cobalt content. However, this comparison cannot be made in A samples because of the possibility of inhomogeneous strains at the grain boundaries of a random polycrystalline material, which is a consequence of the anisotropic magnetostriction in $\mathrm{REFe}_{2}$.

The dependence of $1 / S_{33}^{B}$ on bias field for $A$ and I samples are shown in figures 4 and 5 respectively. An increase in the value of $1 / S_{33}^{B}$ with applied field is observed for I samples of $x=0.0$. The curves for $x=0.5$ and $x=1.2$ show similar behaviour but different from that for $x=0.0$. This may be due to the occurrence of a spin reorientation in $x=0.5$ sample. Rosen et al (1973) observed changes in the absolute values of the elastic constants when the easy direction of magnetization changes from [111] to [100]. Hence the variation of the elastic constants with the applied field may also be dependent on the easy direction of magnetization in these compounds.

The change in the Young's modulus (reciprocal of elastic compliance constant) with applied magnetic field, which is also called $\Delta E$ effect, arises mainly due to domain wall processes during the initial stages of magnetization. A $180^{\circ}$ domain wall motion does not contribute to magnetostriction. $90^{\circ}$ walls are probable when [111] is the easy direction of magnetization whereas $180^{\circ}$ walls could be possible for a [100] direction (Cullity 1972). However, the absolute values of the elastic constants of the lattice are affected by magnetoelastic coupling. 


\section{References}

Abell J S, Butler D, Greenough R D, Joyce V and Pitman K C 1986 J. Mag. Magn. Mater. 626

Abell J S and Lord D G 1986 J. Less Common Met. 126107

Annapoorni S and Rama Rao K V S 1990 J. Appl. Phys. 67424

Belov K P, Kataev G I, Levitin R Z, Nikitin S A and Sokolv' V I 1983 Sov. Phys. USP 26518

Branwood A, Dudley R, Janio A L and Piercy A R 1986 IEEE Bull. 60411

Bushow K H J 1980 Ferromagnetic materials (ed.) E P Wohlforth (Amsterdam: North Holland) Vol. 1

Clark A E 1980 Ferromagnetic materials (ed.) E P Wohlforth (Amsterdam: North Holland) Vol. 1

Cullity B D 1972 Introduction of magnetic materials (Massachussets: Addison Wesley)

Dhilsha K R 1992 Magnetic, magnetomechanical and electrical behaviour of certain compounds of rare-earth transition metals, Ph. D. thesis, I.I.T., Madras

Dhilsha K R, Markandeyulu G and Rama Rao K V S 1991 J. Appl. Phys. 704450

Dhilsha K R and Rama Rao K V S 1990 J. Appl. Phys. 68259

Dhilsha K R and Rama Rao K V S 1993 J. Appl. Phys. 731380

Eriksson O, Johansson B, Brooks M S S and Skriver H L 1989 Phys. Rev. 409519

Jiles D C and Hariharan S 1990 J. Appl. Phys. 675013

Kendall D and Piercy A R 1990 IEEE Trans. Magn. 261837

Korolev A V, Mushnikov N V, Andreyev A V and Gaveko S S 1990 Phys. Met. Metall. 6988

Lee E W and Pourarian F 1976 Phys. Status Solidi A34 383

Oswin J R 1988 Proc. 2nd international conf. on giant magnetostrictive and amorphous alloys for sensors and actuators (Spain: Marbella)

Rosen M, Klimker H, Atzmony U and Dariel M P 1973 Phys. Rev. 82336

Sato K, Isikawa Y, Mori K, Clark A E and Cullen E 1986 J. Mag. Magn. Mater. $54-57875$

Savage H T, Clark A E and Powers J M 1975 IEEE Trans. Mag. 111355

Senthil Kumar M, Reddy K V and Rama Rao K V S 1993 J. Appl. Phys. 74 (to appear)

Sewell J S and Kuhln P M 1988 Power sonics and ultrasonics transducers design (eds) B Harmonic and J N Decarpigney (Berlin: Springer Verlag)

Sherman C H 1975 IEEE Trans. Son. Ultrason. Su-22 281

TERFENOL-D Notes 1990 EDGE Technologies Inc. 3 